\title{
AFFECTIVE SYMPTOMS AND POSTURAL ABNORMALITIES AS PREDICTORS OF HEADACHE: AN APPLICATION OF ARTIFICIAL NEURAL NETWORKS
}

\author{
L. Gitto, G. Massini, F.S. Mennini $\ddagger$ C.Mento§ $\$$ P.M. Buscema ${ }^{\dagger}$
}

\begin{abstract}
Chronic headache is a major liability in the individuals' quality of life. Identifying in advance the main features common to patients with headache may allow planning a preventive strategy of intervention. An artificial neural network model (Auto Contractive Maps - AutoCM), aimed at analyzing the correlations between patients' characteristics, affective symptoms and posture indicators has been developed in this paper. Patients suffering from chronic headache were observed at a neurological centre in Sicily (Italy). Headache and affective states were measured using the Profile of Mood States (POMS), the Beck Depression Inventory (BDI), the Toronto Alexithymia Scale (TAS-20) and the Repression Scale. Postural evaluations were carried through a stabilometric platform. The method of analysis selected allowed to reconstruct some records that were missing, through a Recirculation AutoAssociative Neural Network, and to obtain sound results. The results showed how some items from TAS-20, Repression and POMS were closely linked. The postural abnormalities were correlated primarily with repression features. The highest scores of the POMS were correlated with the items of the BDI. The results obtained lead to interesting remarks about the common traits to patients with headache. The main conclusion lies in the potentialities offered by the new methodology applied, that may contribute, overall, to a better understanding of the complexity of chronic diseases, where many factors concur to define patients' health conditions.
\end{abstract}

Key words: affective symptoms, postural abnormalities, headache, artificial neural networks, auto contractive maps

Received: January 14, 2019

DOI: $10.14311 / \mathrm{NNW} .2020 .30 .001$

Revised and accepted: February 5, 2020

\footnotetext{
*Lara Gitto - Corresponding author; Dipartimento di Economia, Università degli Studi di Messina, Piazza Pugliatti 1, 98122 Messina, Italy, E-mail: Lara.Gitto@unime.it

${ }^{\dagger}$ Giulia Massini; Paolo Massimo Buscema; Semeion, Centro Ricerche di Scienze della Comunicazione, Roma, Italy

‡Francesco Saverio Mennini; CEIS Economic Evaluation \& HTA, Facoltà di Economia, Università di Roma "Tor Vergata", Roma, Italy; Institute for Leadership and Management in Healthcare, Kingston University, London, United Kingdom

${ }^{\S}$ Carmela Mento; Dipartimento di Neuroscienze, Policlinico Universitario di Messina, Messina, Italy
}

(C)CTU FTS 2020 


\section{Background}

Headache, especially when chronic and recurrent, is a major liability in the individuals' quality of life.

Headache disorders vary in severity, incidence and duration: it is possible to distinguish into tension-type headache, migraine and headache caused by medications overuse $^{12}$.

Although headache disorders are among the most common of all health disorders, their epidemiology is only partly documented [5]; it has constituted the object of some reviews, often carried out within international projects.

The Eurolight Project [6] outlined how more than $50 \%$ of adults in European countries declared to have suffered from headache during the past year. Such a widespread prevalence requires a huge amount of economic resources to treat it: hence, chronic headache can be considered one of modern society's most costly illnesses [7].

There are both direct and indirect costs associated to headache and migraine [8]. However, the total costs of headache are not clearly defined $[9,10]$ : indirect costs of migraine often outweigh the direct costs and, therefore, represent an obvious target for healthcare interventions aimed at reducing the burden of migraine [11].

During the last years, several studies on chronic headaches have gone further the analysis of costs, stressing the negative influence of headache on quality of life and on the development of negative emotions $[12,13]$.

Many illnesses are reported to be comorbid with migraine [14], and this circumstance outlines the clinical complexity of this disorder. Moreover, large costs of headache to society have been reported for those patients who have substantial and complicating comorbidities [15]. Cardiovascular disorders coronary heart disease, hypertension, have been recognized as concomitant risk factors for headache [16,17].

Psychiatric disorders, including depression, anxiety disorder, bipolar disorder, and suicide ideation and attempt are frequently comorbid with migraine, and result in a significant burden for patients. Several studies [18] found the prevalence of psychiatric comorbidities in subjects with migraine, determining a poorer healthrelated quality of life [19]; other studies [20] adopted a wider perspective and looked at the cross-sectional associations between pain conditions as migraine, arthritis and back pain, and psychiatric disorders, such as depression, generalized anxiety disorder and panic attacks.

Psychological and affective symptoms considered in the present paper are mainly related to mood states, depression, repression mechanism and alexitmia [21-24].

\footnotetext{
${ }^{1}$ Tension-type headache may be stress-related or associated with musculoskeletal problems in the neck [1]: it pursues a highly variable course, often beginning during the teenage years and reaching peak levels in the 30s.

Migraine can be considered a progressive disease with cardiovascular, cerebrovascular and long term neurologic effects [2]: the World Health Organization now lists migraine among the world's top 20 of all recognized disorders, with an impact that extends far past the suffering individual, to the family and community [3].

Headache caused by medications overuse [4] can be oppressive and persistent: Over time headache episodes become more frequent, as does medication intake and may persists all day.

${ }^{2}$ In spite of the variety of definitions for clinical conditions that are overall similar, in the present paper the terms "migraine" and "headache" will be both used to indicate the main object of investigation.
} 
Gitto L. et al.: Affective symptoms and postural abnormalities as predictors...

Other chronic physical disorders might be associated with migraine. The NordTrøndelag Health Study [25] noted that subjects with headache reported more musculoskeletal pain than other subjects: however, the opposite is true as well, with postural abnormalities more likely to be present in patients who declare to suffer from headache [26].

Large cohort studies are needed to define and confirm the relationship between migraine and its co-morbid diseases, both psychological and physical: the objective is that of providing optimal care, as well as understanding the mechanisms through which migraine is developed.

The presence of affective symptoms (such as depression, altered mood states, alexitimia) and the existence of postural abnormalities may be the signal of latent headache and migraine. Identifying in advance the main features common to patients with headache, as well as the principal risk factors, may allow to plan a preventive strategy of intervention aimed at improving patients' quality of life, together with the reduction in the level of direct and indirect costs [27,28].

In this light, we developed an artificial neural network, with the purpose of analyzing the correlations between patients' characteristics, affective symptoms and physical traits.

The paper is organized as follows: in the next section the clinical factors related to the diagnosis of headache are examined, together with the assessment of affective and physical symptoms.

Then, the modalities through which it is possible to develop a neural network are described; an Auto Contractive neural network (AutoCM) [29] allows to draw a sort of "nervous system" of the data employed, looking at the correlations among the variables and identifying the most significant factors that characterize patients suffering from migraine.

The discussion of the results and some remarks on the potentialities offered by the innovative methodology based on the neural networks, likely to find the "hidden information" within a dataset, conclude the paper.

\section{Methods}

\subsection{Measurement tools}

A survey investigating several aspects (patients' affective symptoms, psychological attitudes and postural assessment) has been administered to a sample of patients suffering from migraine.

The analysis was carried out in 2015 and was related to the patients followed at the IRCCS Centro Studi Neurolesi "Bonino-Pulejo", in Sicily (Italy) diagnosed with migraine: the ethical committee was informed about the intention to create a database containing medical information of patients suffering from headache and related disorders followed at the centre and gave its consent to the studies that could be carried out with the objective to learn more about these patients' characteristics, their epidemiology and pharmacological treatments administered.

Patients were asked to give their consent, both oral and written, to record their personal and clinical information: they were told about the intention to build a 
database containing their characteristics for further investigation on the aetiology of their symptoms and agreed.

Affective symptoms were measured using:

1. The Profile of Mood States (POMS): it is composed of 65 items and employs a five-point rating scale, designed to rapidly assess transient and fluctuating affective states [30]. Six mood states have been derived by factor analysis: Tension-Anxiety (T); Depression-Dejection (D); Anger-Hostility (A); VigorActivity (V); Fatigue-Inertia (F); Confusion-Bewilderment (C).

2. The Beck Depression Inventory (BDI) is a self-report, 21-item questionnaire, originally designed by Beck et al. [31] with the aim of assessing the severity of current depressive symptoms. Responses are assigned a score ranging from 0 to 3 . The total score is the sum of the scores for all answers.

3. Alexithymia is associated with various psychosomatic disorders. It is defined as the difficulty in identifying and distinguishing between feelings and body sensations. The 20-items version of the Toronto Alexithymia Scale (TAS20) [32] contains three subscales that cover: a) difficulty in identifying feelings (7 items), b) difficulty in describing feelings (5 items) and c) externallyoriented thinking (8 items). The scores are derived from a five-point Likert scale and range, overall, from 20 to 100 .

4. The Repression Scale [33] is used to evaluate the conscious mechanism aimed at avoiding stressful stimuli. Higher scores denote greater repression/sensitization.

Instead, postural assessment has been performed through a stabilometric platform.

Many factors, both physical, metabolic and even mental, may determine the posture assumed. The study of posture can give information about the person and his/her physical condition at that time of life.

While, on the one hand, posture has to be regarded as a "static" attitude, with a narrow range of oscillation, on the other hand, the balance is "dynamic", as it requires a series of postures within the projection of the center of gravity to the ground.

In order to work properly, the postural system requires many elements, which are continuously detected by "receptors", such as the neuromuscular spindles, skin receptors, etc. Through the nerve fibers, the receptors send the information to the nervous system, that is transmitted to the "effectors" (i.e. the muscles, which, in turn, receive the input and determine the movement). When one or more receptors fail in sending the correct information, postural imbalances appear.

Together with the receptors, the postural system also uses all the information from other sense organs, as the eye, the ear vestibular apparatus, the sole of the foot and the stomatognathic system.

The eye and the ear (assessed by looking at pupillary symmetry and zygomatic arch) are relevant for the balance with respect to the space. The jaw (evaluated through the mandibular angle and mandibular arrow) performs respiratory function and supports the complex hyoid-tracheal and the position of the skull. The foot 
Gitto L. et al.: Affective symptoms and postural abnormalities as predictors...

(appraised looking at the ankle symmetry) is the last part of the kinetic chain and realizes the maintenance of balance.

The correct posture of the body implies that the individual may stand with his face forward, the upper limbs aligned to the hips and feet wide apart and aligned rear of about $30^{\circ}$.

Through the analysis of the distribution of pressure loads on stabilometric platforms it is possible to discover postural abnormalities. The stabilometric platform, used in many experimental researches on the posture, is equipped with piezoelectric transducers, that can detect the vertical component of the forces exerted on the platform itself. The platform can, therefore, measure the baricentre of the body and its oscillations (stabilometry analysis). Some platforms also allow to register the distribution of the loads on the plantar surface of support (posturometry analysis) [34].

The information related to affective symptoms and postural assessment have been collected and employed in the artificial neural networks model described in the next section.

\subsection{The artificial neural networks}

The correlations among the patients' characteristics, personality traits and musculoskeletal problems can be studied through advanced analysis techniques, such as the use of dynamic mathematical models and artificial neural networks.

Artificial neural networks have received a limited application with regard to healthcare issues: some studies applying this methodology concern Alzheimer disease $[35,36]$ and radiotherapy [37].

More recently, the application of AutoCM and graph theory has been proposed to Sporadic Amyotrophic Lateral Sclerosis [38], and in a study that combines individuals' personality traits with risk factors for coronary stenosis [39].

The architecture of AutoCM was ideated by Massimo Buscema at Semeion Research Center from 2000 to 2007 [29, 40-42].

AutoCM is characterized by a three-layer architecture: an input layer, where the signal is captured from the environment, a hidden layer, where the signal is modulated inside the AutoCM, and an output layer, through which the AutoCM feeds back into the environment based on the stimuli previously received and processed.

Each layer contains an equal number of $\mathrm{N}$ units, so that the whole AutoCM is made of $3 N$ units. The connections between the Input and the Hidden layers are mono-dedicated, whereas the ones between the Hidden and the Output layers are fully connected. Therefore, given $\mathrm{N}$ units, the total number of the connections, $N c$, is given by:

$$
N_{c}=N(N+1) .
$$

All of the connections of AutoCM may be initialized either by assigning the same constant value to each, or by assigning values at random. The best initial value, in practice, is to initialize all the connections with a same, positive value, close to zero.

The learning algorithm of AutoCM may be summarized in a sequence of six characteristic steps: 
1. Signal transfer from the input into the hidden layer;

2. Calculation and accumulation of deltas (weights changes) for each inputhidden connection;

3. Adaptation of the values of the connections between the input and the hidden layers at the end of each epoch;

4. Signal transfer from the hidden into the output layer;

5. Calculation and accumulation of deltas for each hidden-output connection;

6. Adaptation and updating of the value of the connections between the hidden and the output layers at the end of each epoch (a new epoch begins when all the patterns are processed).

Fig. 1 shows the AutoCM architecture.

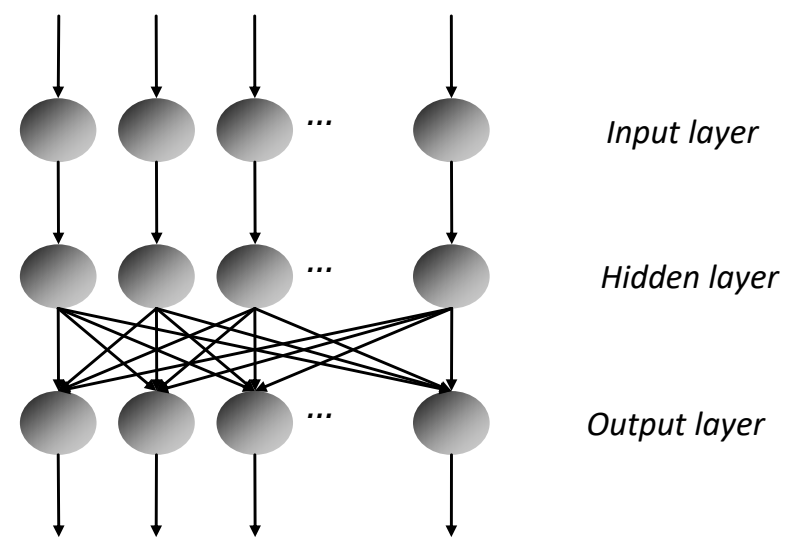

Fig. 1 Architecture of the AutoCM.

The units of the input layer are denoted $m^{[s]}$ (input layer sensors), scaled between 0 and $1, m^{[h]}$ denotes the hidden layer units, and $m^{[t]}$ denotes the output layer units (the system targets). Moreover, we define $v$ to be the vector of the mono-dedicated connections. $W$ denotes the matrix of the connections between the hidden and the output layers where $p$ is the number of the training patterns, $p \in M$ (where $M$ is the total number of the patterns), and $n$ is the epoch number.

In order to specify the steps 1-6 that define the AutoCM algorithm, we have to define the corresponding signal forward-transfer equations and the learning equations, as follows.

a. Signal transfer from the input to the hidden layer:

$$
m_{i, p^{(n)}}^{[h]}=m_{i, p^{(n)}}^{[s]}\left(1-\frac{v_{i(n)}}{C}\right),
$$

where $C$ is a positive real number not lower than 1 . 
Gitto L. et al.: Affective symptoms and postural abnormalities as predictors...

b. The updating of the connections, $v i_{(n)}$, is accomplished through the variation, $\Delta v i_{(n)}$ :

$$
\begin{gathered}
\Delta v_{i(n)}=\sum_{p}^{M}\left(m_{i, p(n)}^{[s]}-m_{i, p(n)}^{[h]}\right)\left(1-\frac{v_{i(n)}}{C}\right) m_{i, p(n)}^{[s]}, \\
v_{i(n+1)}=v_{i(n)}+\alpha \cdot \Delta v_{i(n)},
\end{gathered}
$$

where $\alpha$ is the learning coefficient; $\alpha \geq 1$.

c. Signal transfer from the hidden to the output layer:

$$
\begin{gathered}
\operatorname{Net}_{i, p(n)}=\frac{1}{N} \sum_{j=1}^{N}\left(m_{i, p(n)}^{[h]}\right)\left(1-\frac{w_{i, j(n)}}{C}\right), \\
m_{i, p(n)}^{[t]}=m_{i, p(n)}^{[h]}\left(1-\frac{\operatorname{Net}_{i, p(n)}}{C^{2}}\right) .
\end{gathered}
$$

d. Updating of the connections $w_{i, j(n)}$ through the variation $\Delta w_{i, j(n)}$ :

$$
\begin{gathered}
\Delta w_{i, j(n)}=\sum_{p}^{M}\left(m_{i, p(n)}^{[h]}-m_{i, p(n)}^{[t]}\right)\left(1-\frac{w_{i, j(n)}}{C}\right) m_{j, p(n)}^{[h]} \\
w_{i, j(n+1)}=w_{i, j(n)}+\alpha \cdot \Delta w_{i, j(n)}
\end{gathered}
$$

During the "training" performed in each period, in addition to the calculation of the output values, the algorithm calculates the correction quantity of the input weights.

At the end of the learning process, all the information is transferred to the matrix of connections $w$. This matrix represents therefore the AutoCM knowledge about the whole data set.

The matrix $w$ is a square matrix which shows both on the rows and on the columns the correlations between the variables. The higher the value of each connection, the greater the correlation between these variables.

To better appreciate the results of this process two types of graphs can be employed: the Minimum Spanning Tree (MST) and the Maximally Regular Graph (MRG, Semeion(C). Both MST and MRG are special graphs, capable to read the main information from the weights matrix, generated by AutoCM during the training phase.

The MST might be called the nervous system, or minimal structure system, of any dataset. Indeed, adding up the connections selected by the MST, we obtain the total energy of the system.

The MST selects only those connections that minimize this energy, in other words, the only connections that are really necessary to maintain a cohesive system.

The main limit of MST is inherent to its very nature: each connection that originates an internal cycle in the graph is eliminated, regardless of its strength and significance.

In order to solve this problem and to better capture the inherent complexity of a dataset, it is necessary to add more connections to the MST, on the basis of 
two criteria: the new connections must be relevant from a quantitative point of view and must be able to generate new cyclic microstructures that highlight the regularities in the data set from a qualitative point of view.

In this way, the MST may be transformed into an undirected cyclic graph, representing a dynamic system with a temporal dimension. This new graph should provide information not only on the structure, but also on the functions of the dataset variables.

The ordered list of connections that were skipped as the MST was being generated have to be considered; a function $H_{0}$, obtained each time a new connection is added to the basic MST structure, so that the variation in the complexity of the graph is monitored is estimated.

The Maximally Regular Graph (MRG) is that graph whose function $H_{0}$ reaches the highest value among all the graphs generated by adding to the original MST the most relevant dataset connections. The higher the value of the function $H_{0}$, the more significant is the cyclic microstructure predicted by its graph (see Appendix for a more detailed description).

Overall, through MST and MRG, it is possible to represent and analyze the connections among input variables.

Here, the connections among variables related to patients' psychological assessment and posture have been studied to verify to what extent headache and related disorders may be due to the patients' psychological traits or to a correct posture.

\section{Results}

Forty-four patients diagnosed with headache, according to the Headache Classification criteria established by the International Headache Society [43], were included in the observed sample.The majority of patients in the sample were female $(35$ subjects $=69.55 \%)$. They were aged between 15 and 67 years old $($ mean $=35.4$; S.D. $=13.92)$; almost $45.50 \%$ of patients were younger than 30 years old). About $64 \%$ completed higher education, while $16 \%$ completed university education.

Some statistics can be observed in Tab. 1.

\begin{tabular}{ccccc}
\hline Variable & Mean & Standard Deviation & Min & Max \\
\hline Gender & 0.204 & 0.408 & 0 & 1 \\
Age & 35.432 & 13.924 & 15 & 67 \\
< 30 years & 0.454 & 0.504 & 0 & 1 \\
30 - 50 years & 0.364 & 0.487 & 0 & 1 \\
> 50 years & 0.182 & 0.390 & 0 & 1 \\
Primary education & 0.204 & 0.409 & 0 & 1 \\
Higher education & 0.636 & 0.487 & 0 & 1 \\
Graduate & 0.159 & 0.370 & 0 & 1 \\
\hline
\end{tabular}

Tab. I Sample descriptive statistics. 
Gitto L. et al.: Affective symptoms and postural abnormalities as predictors...

Data obtained from the scales aimed at assessing affective symptoms (POMS, BDI, TAS-20, Repression Scale) can be seen in Tab. II, that allows to appreciate the richness of information collected, related to the items of each scale.

\begin{tabular}{|c|c|c|c|c|}
\hline Variable & Mean & Std. Dev. & Min & $\operatorname{Max}$ \\
\hline \multicolumn{5}{|l|}{ Profile of Mood States (POMS) } \\
\hline Tension-Anxiety (T) & 58.522 & 11.205 & 43 & 85 \\
\hline Depression-Dejection (D) & 52.568 & 12.652 & 40 & 89 \\
\hline Anger-Hostility (A) & 55.909 & 15.011 & 40 & 99 \\
\hline Vigor-Activity (V) & 50.772 & 12.013 & 27 & 73 \\
\hline Fatigue-Inertia (F) & 57.750 & 12.935 & 38 & 92 \\
\hline Confusion-Bewilderment (C) & 53.250 & 10.760 & 37 & 79 \\
\hline \multicolumn{5}{|l|}{ Beck Depression Inventory (BDI) } \\
\hline Total score & 10.750 & 7.647 & 1 & 30 \\
\hline Sadness & 0.591 & 0.725 & 0 & 2 \\
\hline Pessimism & 0.363 & 0.685 & 0 & 3 \\
\hline Past failures & 0.341 & 0.608 & 0 & 2 \\
\hline Loss of pleasure & 0.591 & 0.757 & 0 & 3 \\
\hline Guilty feelings & 0.500 & 0.821 & 0 & 3 \\
\hline Punishment feelings & 0.204 & 0.594 & 0 & 3 \\
\hline Worthlessness & 0.227 & 0.605 & 0 & 3 \\
\hline Self-criticalness & 0.795 & 0.954 & 0 & 3 \\
\hline Suicidal thoughts or wishes & 0.159 & 0.526 & 0 & 3 \\
\hline Crying & 0.454 & 0.875 & 0 & 3 \\
\hline Irritability & 0.841 & 0.888 & 0 & 3 \\
\hline Loss of interest & 0.318 & 0.561 & 0 & 2 \\
\hline Indecisiveness & 0.773 & 0.911 & 0 & 2 \\
\hline Self-dislike & 0.386 & 0.784 & 0 & 3 \\
\hline Concentration difficulties & 0.773 & 0.886 & 0 & 3 \\
\hline Change in sleep patterns & 0.932 & 0.818 & 0 & 3 \\
\hline Tiredness and/or fatigue & 1.022 & 0.698 & 0 & 3 \\
\hline Change in appetite & 0.273 & 0.499 & 0 & 2 \\
\hline Weight loss (loss of energy) & 0.341 & 0.834 & 0 & 3 \\
\hline Agitation & 0.636 & 0.809 & 0 & 3 \\
\hline Change libido & 0.273 & 0.660 & 0 & 3 \\
\hline Beck 1 (no or mild depression) & 0.704 & 0.461 & 0 & 1 \\
\hline Beck 2 (moderate depression) & 0.136 & 0.347 & 0 & 1 \\
\hline Beck 3 (severe depression) & 0.160 & 0.370 & 0 & 1 \\
\hline \multicolumn{5}{|l|}{ Toronto Alexithymia Scale (TAS 20) } \\
\hline TAS 1 - Confusion about emotions & 2.295 & 1.391 & 0 & 5 \\
\hline TAS 2 - Difficulty to find the right words & 2.681 & 1.762 & 0 & 5 \\
\hline TAS 3 - Physical sensations not recognizable & 1.931 & 1.283 & 0 & 5 \\
\hline TAS 4 (inverse scoring) - Description of feelings & 3.750 & 1.511 & 0 & 5 \\
\hline TAS 5 (inverse scoring) - Looking in depth at problems & 3.681 & 1.491 & 0 & 5 \\
\hline TAS 6 - Difficulty in identifying emotions when anxious & 2.795 & 1.651 & 0 & 5 \\
\hline TAS 7 - Confusion about body sensations & 2.159 & 1.478 & 0 & 5 \\
\hline TAS 8 - Refusal to understanding & 2.227 & 1.428 & 0 & 5 \\
\hline TAS 9 - Difficulty in identifying feelings & 1.841 & 1.238 & 0 & 5 \\
\hline TAS 10 (inverse scoring) - Need to know emotions & 4.136 & 1.357 & 0 & 5 \\
\hline TAS 11 - Difficulty in describing feelings towards other people & 1.954 & 1.311 & 0 & 5 \\
\hline TAS 12 - Being requested to speak more about themselves & 2.045 & 1.397 & 0 & 5 \\
\hline TAS 13 - Confusion about how things are going on & 2.318 & 1.537 & 0 & 5 \\
\hline TAS 14 - Unjustified anger & 2.750 & 1.644 & 0 & 5 \\
\hline TAS 15 - Avoiding talking about other people's feelings & 2.841 & 1.524 & 0 & 5 \\
\hline TAS 16 - Seeking for entertainment & 2.772 & 1.669 & 0 & 5 \\
\hline TAS 17 - Difficulty in talking even with closest friends & 2.704 & 1.786 & 0 & 5 \\
\hline
\end{tabular}


TAS 18 (inverse scoring) - Empathy

$\begin{array}{llll}3.977 & 1.422 & 0 & 5\end{array}$

TAS 19 (inverse scoring) - Self-examination

$3.477 \quad 1.606$

TAS 20 - Movies, dramas interpretation

Total score

$\begin{array}{llll}45.681 & 15.496 & 0 & 71\end{array}$

Alexitimia $($ yes $=1 ;$ no $=0$ )

0.250

Repression Questionnaire (Likert Scale: $0=$ absolutely true; $5=$ absolutely false) RS 1: Emotion control

$3.114 \quad 1.350 \quad 0$

RS 2: Hiding joy

RS 3: Repression of negative thoughts

$\begin{array}{llll}2.204 & 1.593 & 0 & 5\end{array}$

RS 4: Brooding and recalling thoughts (inverse scoring)

$2.432 \quad 1.485 \quad 0 \quad 5$

RS 5: Ideation (inverse scoring)

$\begin{array}{llll}3.068 & 1.590 & 0 & 5\end{array}$

RS 6: Daydreaming (inverse scoring)

RS 7: Positive thoughts to sleep

RS 8: Simulating disgust

RS 9: Reaction Control

RS 10: Headache

RS 11: Psychological Repression

$2.841 \quad 1.493$

$2.818 \quad 1.646$

$2.545 \quad 1.606$

$1.841 \quad 1.328$

$3.160 \quad 1.363$

$1.614 \quad 1.466$

$2.227 \quad 1.507$

RS 12: Morality

RS 13: Religiosity (inverse scoring)

$3.614 \quad 1.588$

$2.931 \quad 1.662$

RS 14: Sexual rigid education

RS 15: Agitation and calm

$3.160 \quad 1.641$

$2.818 \quad 1.402$

RS 16: Imagination

RS 17: Repressing anxiety

RS 18: Repressing thoughts (inverse scoring)

RS 19: Repressing musical motives (inverse scor

$2.454 \quad 1.438$

$2.682 \quad 1.394$

$\begin{array}{ll}3.023 & 1.732\end{array}$

RS 20: Deception victim when child

$2.660 \quad 1.478$

RS 21: Rationality

$3.364 \quad 1.349$

$2.660 \quad 1.509$

RS 22: Neglecting dreams

$3.00 \quad 1.540$

$2.660 \quad 1.493$

RS 24: Self control

$\begin{array}{ll}3.091 & 1.411 \\ 3.409 & 1.545\end{array}$

RS 25: Removal of impure thoughts

$2.250 \quad 1.432$

RS 27: Trasgression

$\begin{array}{ll}2.250 & 1.432 \\ 2.590 & 1.245\end{array}$

$2.295 \quad 1.357$

RS 28: Free thought from emotions

RS 30: Removal of unacceptable wishes

$\begin{array}{llll}2.750 & 1.542 & 0 & 5\end{array}$

Tab. II Affective symptoms descriptive statistics (POMS - 6 items, BDI - 21 items, 3 levels depression, TAS-20 - 20 items and total score -, Repression - 30 items).

About the POMS, the maximum average value pertained to $\mathrm{T}$, while the lowest values reported (both minimum and maximum) were associated to $\mathrm{V}$.

The total score obtained through the administration of BDI ranged from 1 to 30. It is possible to group patients according to their level of depression: patients with no or minimum level of depression belong to group 1 (Beck 1); patients with moderate depression were grouped in the second group (Beck 2); pathological depression characterizes patients belonging to the third group (Beck 3). The majority of patients $(70.45 \%)$ were included in the first group.

About alexithymia, a cut-off score of $<50$ was used to design a state of nonalexithymia, measured through the TAS-20 scale. In the observed sample this condition was observed for the $25 \%$ of patients. 
Gitto L. et al.: Affective symptoms and postural abnormalities as predictors...

Regarding the Repression Scale, the highest scores concerned items 12, 14 and 1 , related to emotion control, morality and sexual education, while the lowest score was recorded for item 8 , concerning the simulation of disgust.

Results of the assessments carried out with the stabilometric platform, evaluated by physicians and health personnel, are reported in Tab. III.

\begin{tabular}{lccccc}
\hline Variable & $\begin{array}{c}\text { No. of } \\
\text { observations }\end{array}$ & Mean & $\begin{array}{c}\text { Standard } \\
\text { deviation }\end{array}$ & Min & Max \\
\hline Structural index & 34 & 1769.088 & 94.737 & 1604 & 1973 \\
Bi-pupillar symmetry & 41 & 1.536 & 2.169 & 0 & 7 \\
Zygomatic symmetry & 34 & 2.118 & 1.935 & 0 & 8 \\
Right mandibular angle & 28 & 55.857 & 7.541 & 42 & 69 \\
Left mandibular angle & 29 & 55.379 & 12.483 & 32 & 78 \\
Mandibular arrow & 7 & 51.286 & 30.302 & 6 & 100 \\
Hindfoot pronation & 2 & 2.000 & 2.828 & 0 & 4 \\
Supra foot pronation & 2 & 5.500 & 4.948 & 2 & 9 \\
Arrows retro-version & 24 & 101.250 & 24.235 & 60 & 180 \\
Shoulders symmetry & 34 & 1.676 & 1.036 & 0 & 4 \\
Scapular symmetry & 43 & 2.139 & 1.781 & 0 & 6 \\
Dorsal arrow & 25 & 18.88 & 20.559 & 0 & 93 \\
Lumbar arrow & 24 & 58.25 & 14.326 & 33 & 83 \\
Dorsal neck angle & 43 & 2.093 & 1.716 & 0 & 7 \\
Lumbar neck angle & 44 & 2.023 & 1.606 & 0 & 6 \\
Pelvic symmetry & 42 & 1.500 & 1.864 & 0 & 10 \\
Right anteversion pelvis & 27 & 18.518 & 15.260 & 2 & 84 \\
Left anteversion pelvis & 26 & 17.461 & 7.601 & 6 & 29 \\
Posterior superior iliac & & & & & \\
spines (S.I.P.S.) symmetry & 44 & 2.318 & 2.457 & 0 & 15 \\
Patellar symmetry & 3 & 1.667 & 1.548 & 1 & 3 \\
Ankle symmetry & 2 & 1.500 & 2.121 & 0 & 3 \\
Kyphosis arrow & 6 & 62.667 & 20.636 & 43 & 97 \\
Dorsal curve & 42 & 2.00 & 1.623 & 0 & 7 \\
Lumbar curve & 42 & 1.976 & 1.615 & 0 & 6 \\
Beck degrees & 1.454 & 0.761 & 1 & 3 \\
\hline
\end{tabular}

Tab. III Stabilometric platform evidence (25 variables).

More than 100 variables, concerning psychological and postural assessments, were employed in the analysis.

The variables are sub-divided into macro-topics as follows:

- 8 variables relate to patients' characteristics: gender, age (under 30 years old, $31-50$ years old, over 50 years old), education (primary, higher, college education);

- 15 variables relate to POMS items: while for T, D and A two different levels 
have been considered (higher than 60 and normal: within 40 and 60 ), the remaining items $(\mathrm{V}, \mathrm{F}$ and $\mathrm{C})$ present three levels $(<40,40-60$ and $>60)$;

- 24 variables relate to BDI (21 items +3 levels of cut-off);

- 22 variables relate to TAS-20 (20 items + a dummy variable indicating the presence of alexitimia or no);

- 30 variables related to the Repression Scale;

- 15 variables related to the assessments made with the stabilometric platform.

However, while data related to general information, POMS, BDI, TAS-20, Repression Scale, were available for all 44 patients, information related to postural abnormalities were complete only for some variables (lombar neck angle and Posterior Superior Iliac Spines - S.I.P.S. - symmetry). Many of the other data gathered through the stabilometric platform were missing and had to be re-constructed.

A data elaboration with artificial neural networks systems can be reliable if the database to be analyzed and its records are comprehensive and complete. Then, if the incomplete records had to be eliminated, the resulting database would not be representative.

In order to reconstruct the missing data, different methodologies can be used. In this paper, a Recirculation AutoAssociative artificial neural networks has been considered as reconstructive methodology of the missing data [44].

Several steps have been followed.

Step 1. Two samples have been chosen (let us call them A and B): sample A consisted of 24 complete records; sample B consisted of 20 incomplete records of data relevant to the "postural abnormalities".

Step 2. Sample A was employed to train the AutoAssociative artificial neural networks. The network was composed of 114 input nodes (101 complete variables and 13 incomplete variables). Once the learning process occurred, the weights matrix was memorized.

Step 3. The memorized weights matrix was used to "ask" the network about the sample B, establishing as independent variables the 101 relevant to the complete variables, and, as dependent variables, the 13 relevant to the "postural abnormalities". The re-entry technique was used [45]. In this way, the system was able to reconstruct the data of the 13 out of 15 independent incomplete variables.

The description of the 115 variables considered in constructing the artificial neural network is reported in Tab. IV. Further details about AutoCM and the graphs that can represent it (MST and MRG) are given in the Appendix.

The MST related to 44 subjects included in the sample can be observed in Fig. 2.

In order to obtain this graph, the MST selects the most significant relations between the units from the weights matrix W (i.e. between the Hidden units and the output units, see Tab. V) within the AutoCM network, and creates a tree 


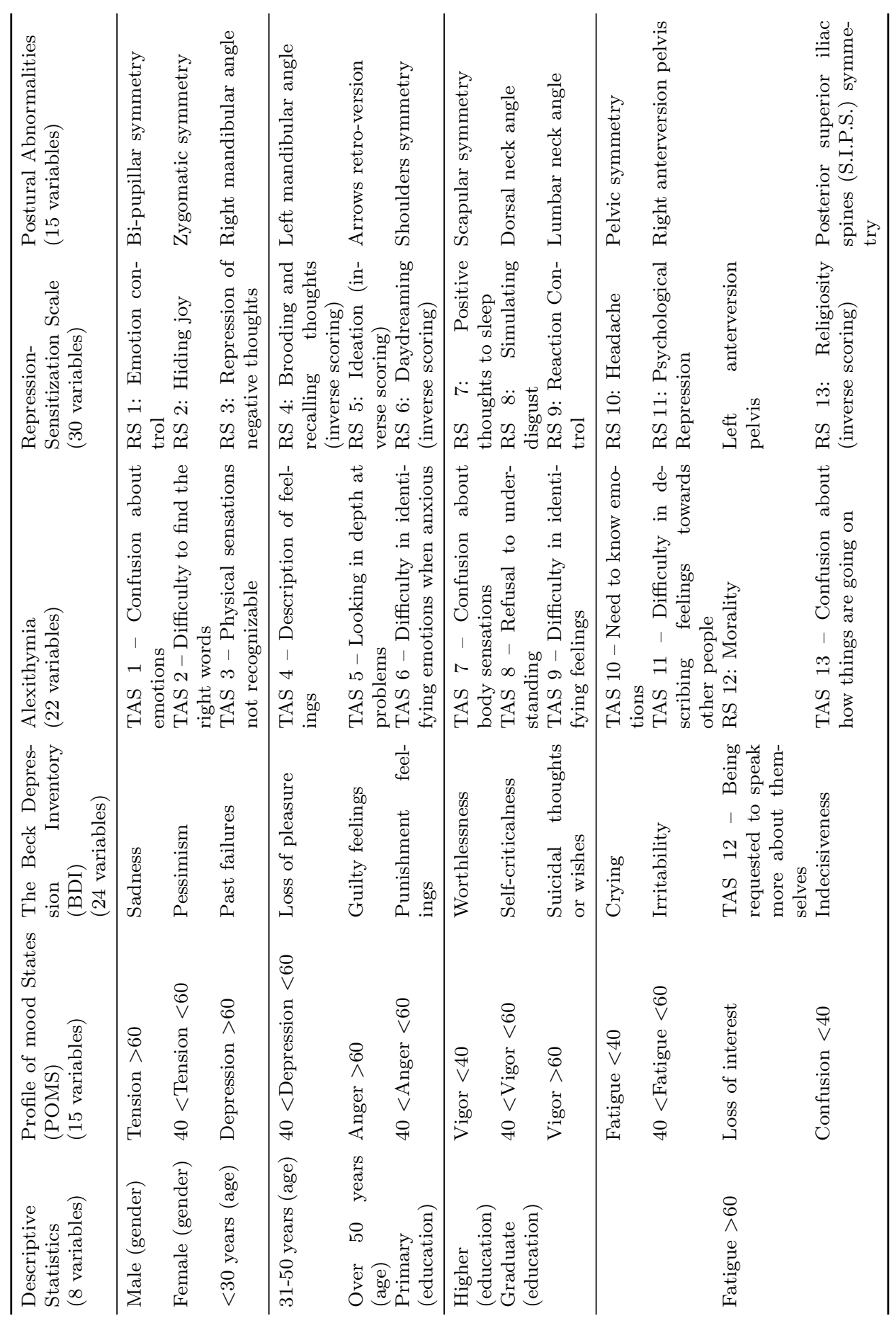




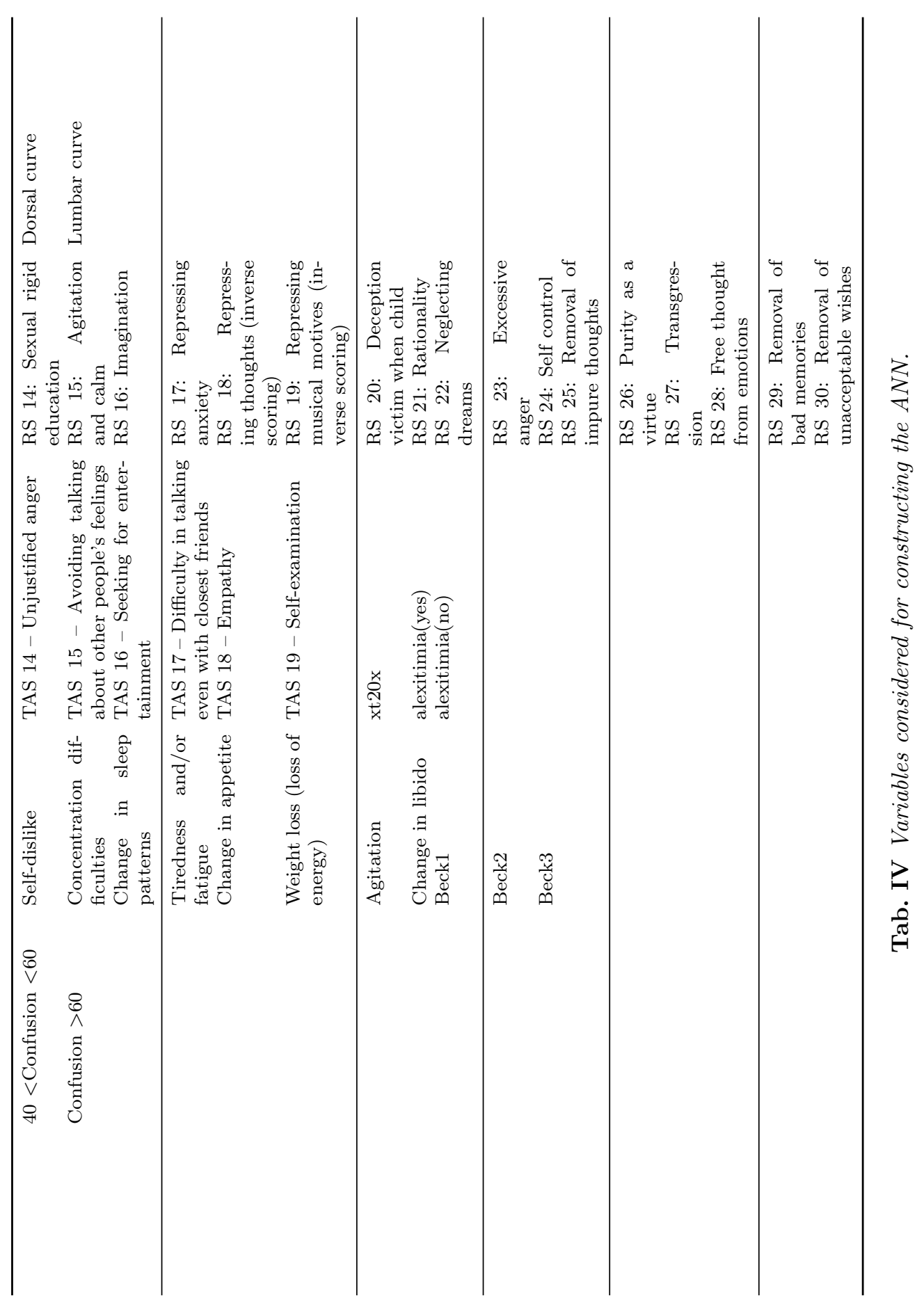


Gitto L. et al.: Affective symptoms and postural abnormalities as predictors...

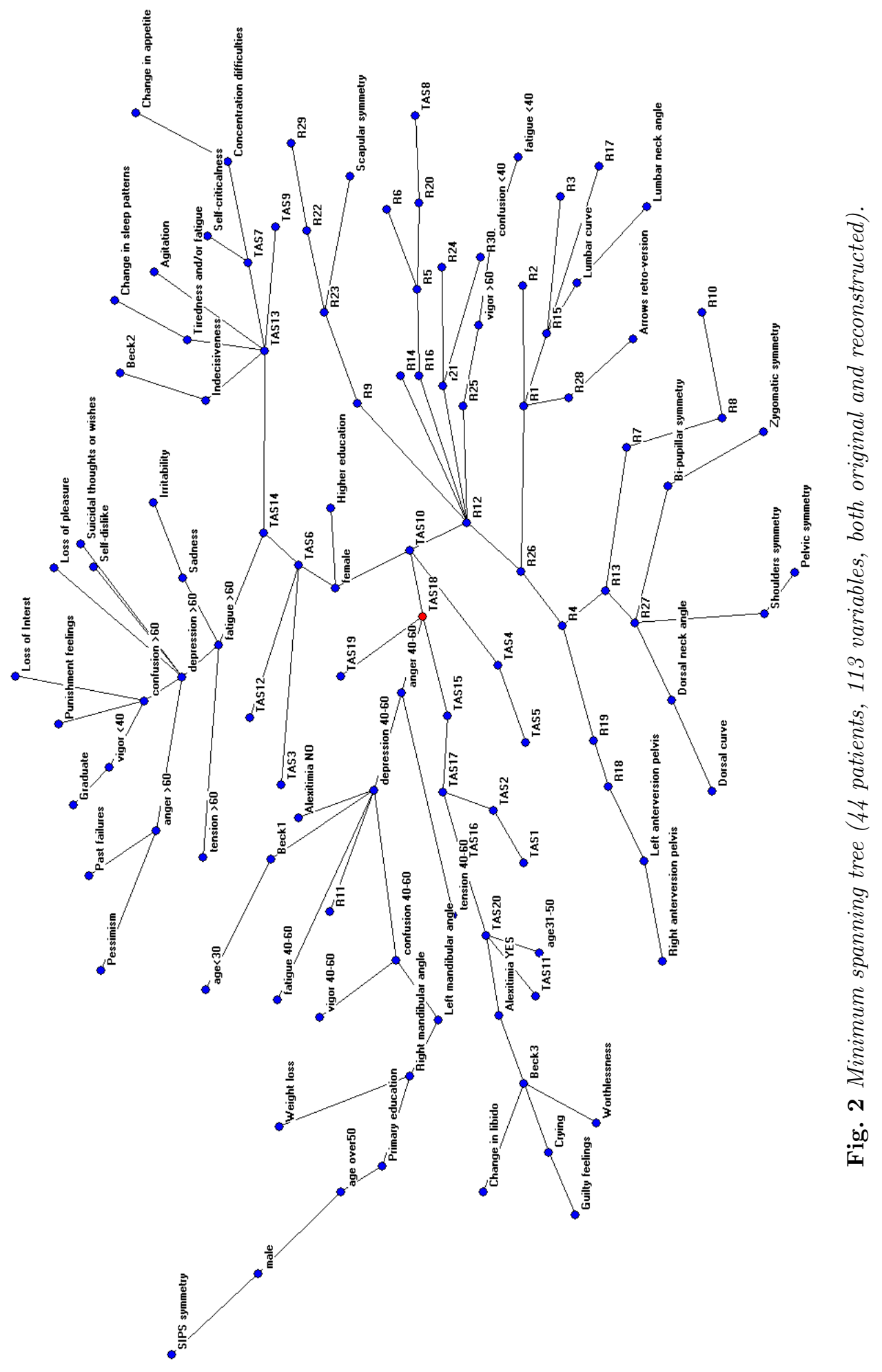


where the branches represent the value of weights. When two variables are diretly connected by a branch, it means that the relation between the variables, that behave in a similar way, is very strong with respect to the whole economy of the dataset.

We show, in Fig. 3, a part of the $\mathrm{W}$ matrix, that outline the relation between the Hidden units and the Output units produced by this process.

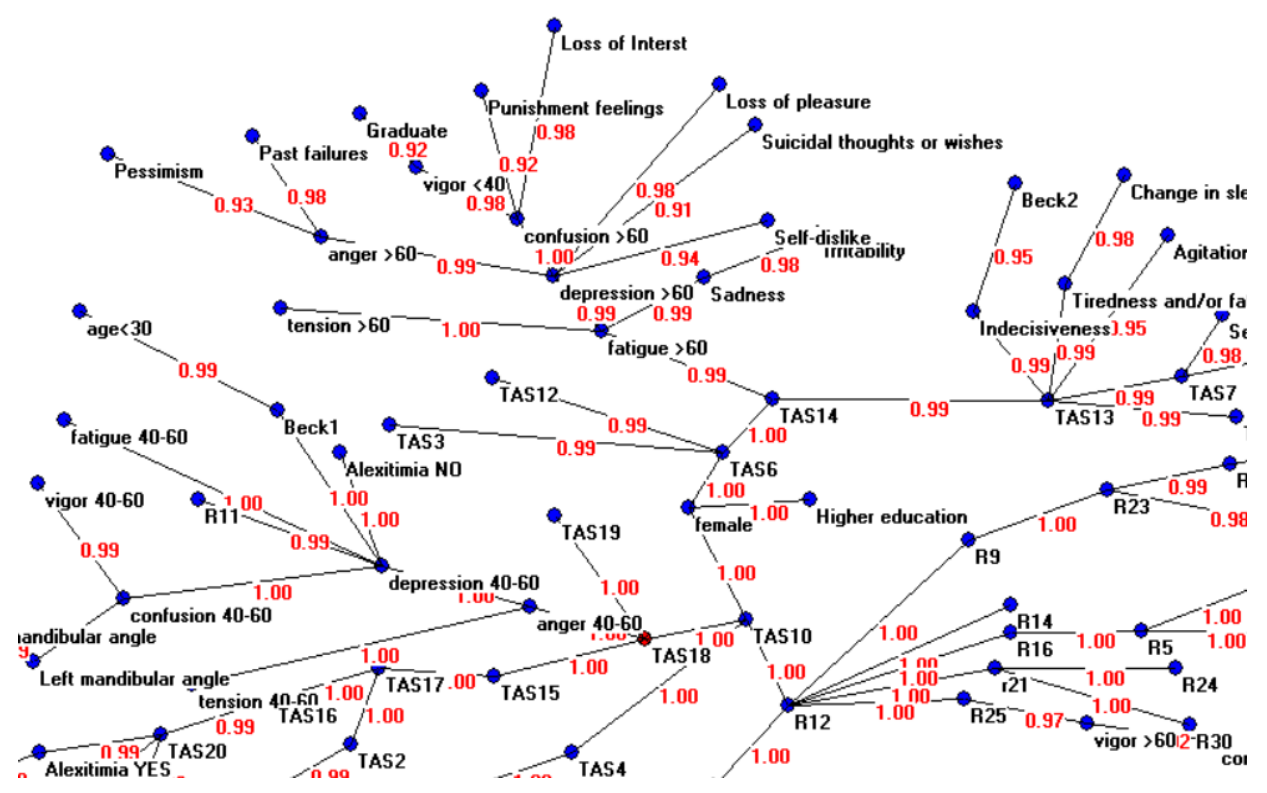

Fig. 3 Connections highlighted by the $M R G$.

As it is possible to see, the network has assigned to each pair of variables a value, that is stronger the greater the relationship existing between the two variables.

In Tab. V it can be seen, for example, a relation with value 0.99 between "male" and "age over 50" which we observe in the graph in Fig. 2 in the form of a branch connecting "male" to the variable "age over 50".

For sake of clarity, in Tab. V it is possible to observe 23 of the 114 input units; the relations selected by the MST, that constitute the branches of the graph, have been highlighted.

Fig. 4 shows, instead, the MRG.

As it is possible to see, the MRG added further connections to the MST graph.

Item 18 of TAS appears as the principal hub of the variables network. Other dominant variables are the scores for TAS-10, RS12 and RS26.

From the graph it appears how the items belonging to the same questionnaires group together: this circumstance has been observed, for example, for TAS items, the Repression Scale and many items of the BDI. Postural abnormalities are mainly related to the Repression Scale items.

The MRG outlined those items whose connections are tighter: all of them refer to mental health dimension rather than physical factors.

Hence, mental health factors play a relevant role in causing headache, rather 
Gitto L. et al.: Affective symptoms and postural abnormalities as predictors...

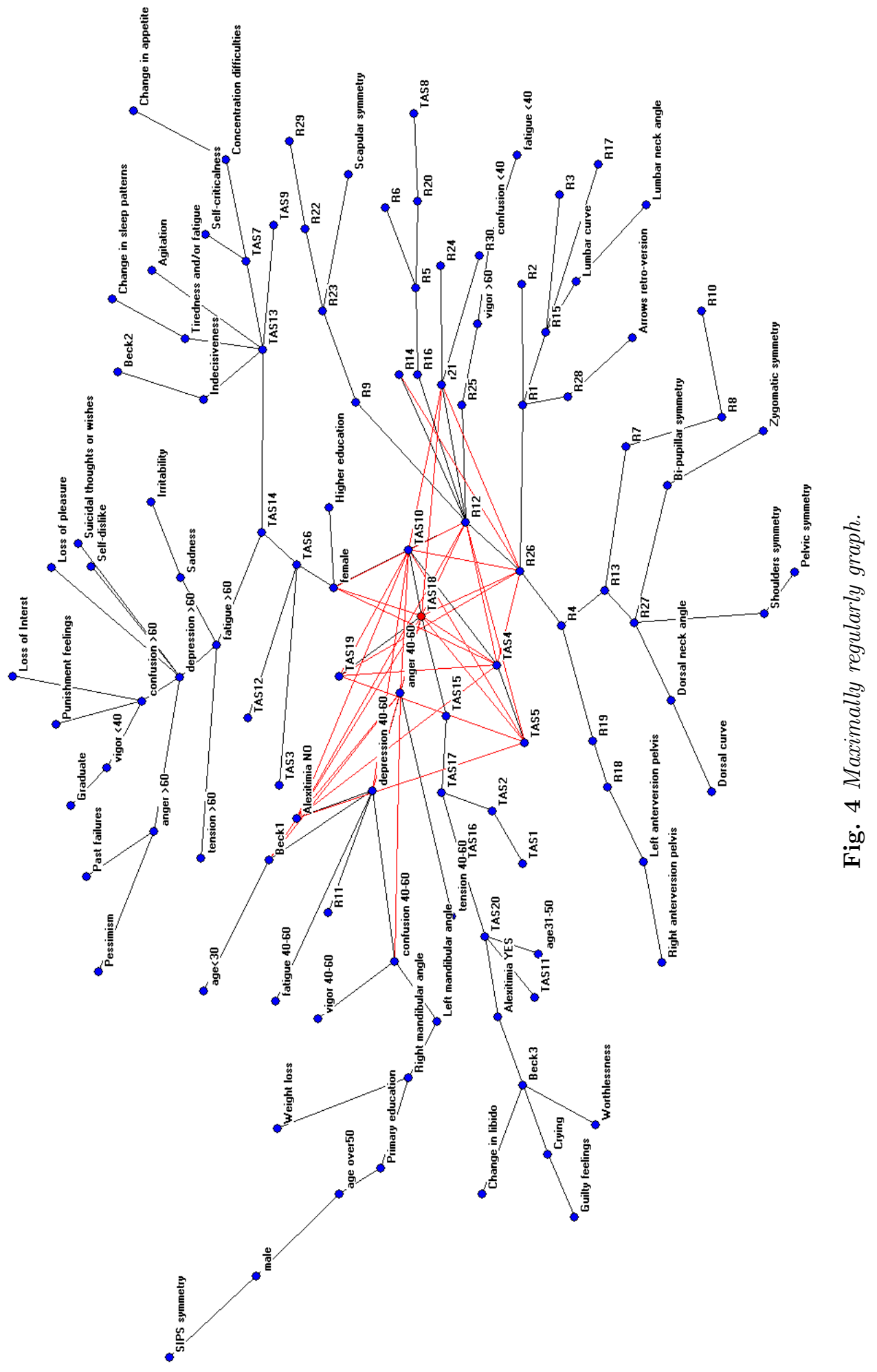




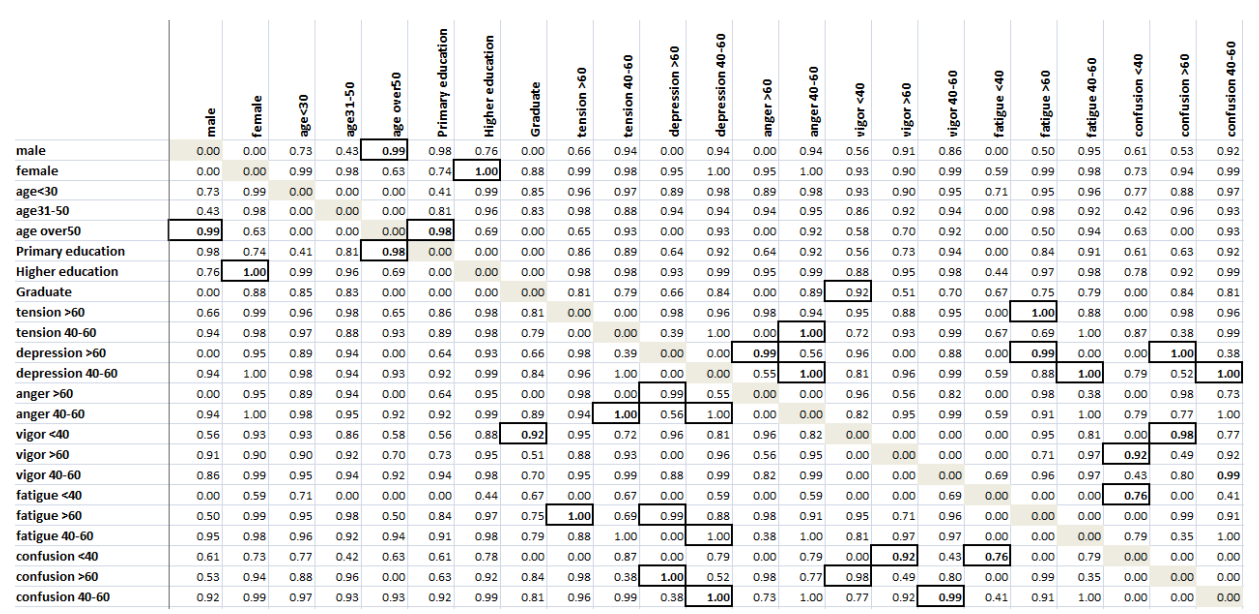

Tab. $\mathbf{V}$ Values of the matrix $W$ of the relations between the units of the Hidden level and the Output level of the AutoCM network after learning.

than postural abnormalities, that are associated, instead to a binding and self repressive attitude.

\section{Discussion}

The MST and MRG show how some items of the TAS-20 and the Repression Scale, together with some intermediate values of the constructs measured by the POMS and related to depression, anger and fatigue are closely related to each other.

This finding is plausible and confirms how the three components of the affectiveemotional sphere (emotional factors, alexythimia and repression of emotions) are interconnected among them. Especially the items relating to the Repression Scale tend to aggregate together.

The item 12 ("I consider myself a person with a clear moral") and 26 ("Purity is a virtue") are connected with some items of the TAS-20 and to the rest of the network. In particular, the item R12 is correlated both with the item 10 of the TAS-20 ("It is essential to know my own emotions") and with the item 18 ("I can feel close to someone, even if it happens that we are silent"); the latter connects with item 4 ("I can easily describe my feelings").

This evidence outlines how the assessment of alexithymia, concerning especially items 4, 10 and 18, that represent the less "pathological" items, correlates with the moral dimension of each individual. Such dimension focuses on the attention to the individual's social behavior and is based on the openness towards other people: the psychological attitude is opposite to introverted mood and the auto-creation of psychosomatic symptoms.

The only item of the Repression Scale differing from the others is item 11 " $I$ often remind scenes that can easily go out of my head", correlated only with a moderate level of depression. This circumstance is understandable, because this item reflects a mental operation that involves rational mechanisms. When used 
Gitto L. et al.: Affective symptoms and postural abnormalities as predictors...

excessively, the latter contributes to the strengthening of the depressive symptom: in particular, the rationalization processes are usually found in clinical depression.

Postural abnormalities are related primarily to the Repression questionnaire: item 27 ("I like to transgress with the mind") is related to abnormalities of the spine, pupillary symmetry and symmetry of the shoulders.

Postural abnormalities are linked together, so that it is possible, in several cases, to assume a consequentiality between some anomalies (i.e. pupillary symmetry and zygomatic symmetry; lumbar curve with lumbar neck angle and dorsal curve).

The highest values of the POMS are connected with items of the BDI. This result is plausible as well, as alterations of the affective state are often coupled with variations of individuals' emotional state.

Variables related to gender, age and education level are rarely correlated with psychological symptoms, except for graduate education, which is connected to a state of diminished energy, as well as confusion, depression and tiredness. It could be inferred how, in order to reach high levels of education, people invest primarily in mental effort, repressing the emotional dimension, with the consequence that affective symptoms are manifested as psychosomatics.

Postural asymmetries related to mandibular angle, both on the right and left hand side, are connected to weight loss: in other words, postural abnormalities determine other anomalies at the psychological and physical level, as the loss of appetite or the decrease in weight. The latter could be the consequence of a physical abnormality that leads to the difficulty in chewing and the subsequent difficulty in the ingestion of solid foods.

Gender differences do not seem to have a key role in the definition of postural abnormalities: observing the MRG, it can be noticed how the female gender is closely related with some items of TAS-20 (for example, item 10: "It is essential to know your own emotions", or item 6: "When I am upset I do not know if I'm sad, scared or angry").

Women typically pay more attention to emotional states and loss of control with respect to them; they are, indeed, more prone to anxiety.

The male gender, instead, is correlated with old age and abnormalities in S.I.P.S.

\section{Conclusions}

In this paper we have presented the mathematical algorithms that lead to the development of a new artificial neural networks, namely the AutoCM.

AutoCM reshapes the distances among variables or records of any dataset, considering their global vectorial similarities.

The artificial adaptive system, represented through the MST, is likely to define the strength of the associations of each variable with all the other in a dataset: it shows visually the map of the main connections of the variables and the basic semantic of their ensemble.

The MRG shows, instead, which connections are the strongest.

The results obtained lead to interesting remarks: the network constructed with the data collected on a sample of patients suffering from headache matches most of the clinical and pathological features commonly recognized by experts in this field. 
Hence, AutoCM allows to identify the emotional and psychological factors that should be taken into consideration when developing a therapeutic plan for patients suffering from migraine (the psychological aspects of repression and alexythimia for example).

This methodology may contribute to a better understanding of the complexity of the disease, allowing, in this way, to "personalize" medical treatments according to patients' characteristics.

The analysis has been limited to a cohort of patients from one medical centre only. It might be replicated widening the sample to check if the results may be generalized.

The resulting evidence could be examined jointly by physicians (neurologists, physiatrists) and psychologists, who could therefore appreciate the relevance of each item in framing the diagnosis and allowing to plan an therapeutic program.

Finally, clinical guidelines to tackle both medical and psychological aspects could be developed.

Overall, the results confirm the complexity of the disorder and the variety of aspects that should be taken into consideration when planning an adequate assistance.

\section{Acknowledgement}

The authors would like to thank Dr. Rosario Grugno, responsible of the ambulatory for headache and migraine at the IRCCS Centro Neurolesi "Bonino Pulejo" (Messina, Italy), Prof. Salvatore Settineri, who developed the Repression Scale and Dr. Antongiulio Vitetta, who contributed to collect data. They remain solely responsible for every error. This research project has not benefited by any funding.

\section{Conflict of interest None.}

\section{References}

[1] SOHN J.H., CHOI H.C., LEE S.M., JUN A.Y. Differences in cervical musculoskeletal impairment between episodic and chronic tension-type headache. Cephalalgia, 2010, 30, pp. $1514-23$.

[2] HAZARD E., MUNAKATA J., BIGAL M.E., RUPNOW M.F.T., LIPTON R.B. The Burden of Migraine in the United States: Current and Emerging Perspectives on Disease Management and Economic Analysis. Value in Health, 2009, 12, pp. 55-64.

[3] ROSS S. The Astronomical Costs Of Migraine, World Headache Alliance, http://www. headache-help.org/free-articles-headaches. Accessed 1 October 2014.

[4] Curone M., Tullo V., LOVATi C., PROIETti CECChini A., D’AMiCO D. Prevalence and profile of obsessive-compulsive trait in patients with chronic migraine and medication overuse. Neurological Sciences, 2014, 35(S1), pp. 185-187,

[5] World Health Organization. World health report 2001. WHO, Geneva, 2001.

[6] STOVNER L.J., ANDRÉE C. Prevalence of headache in Europe: a review for the Eurolight project. Journal of Headache and Pain, 11pp, 2010, pp. 289-299.

[7] LANTERI-Minet, M. Economic Burden and Costs of Chronic Migraine. Current Pain Headache Report, 2014, 18, pp. 385, doi: 10.1007/s11916-013-0385-0. 
Gitto L. et al.: Affective symptoms and postural abnormalities as predictors...

[8] STOVNER L.J., ANDRÉE C. Impact of headache in Europe: a review for the Eurolight project. Journal of Headache and Pain, 2008, 9, pp. 139-146.

[9] MENNINI F.S., GITTO L., MARTELLETTI P. Improving care through health economics analyses: cost of illness and headache. Journal of Headache and Pain, 2008, 9, pp. 199-206.

[10] MENNini F.S., GiTTO L., MARCELlUSi A., SAKAi F., ARAKAWA I. Economics of Headache, in Martelletti P. and Steiner T.J. (eds.). Handbook of Headache: Practical Management, Springer, 2011.

[11] SOLOMON G.D., PRICE K.L. Burden of migraine. A review of its socioeconomic impact. Pharmacoeconomics, 1997, 11(S1), pp. 1-10.

[12] BLUMENFELD A. Botulinum toxin type A treatment of disabling migraine headache: a randomised double-blind, placebo controlled study. Headache, 2003, 43, pp. pp. 853-860.

[13] YANG G., BAAD-HANSEN L., WANG K., XIE Q.F., SVENSSON P. Effect of negative emotions evoked by light, noise and taste on trigeminal thermal sensitivity. Journal of Headache and Pain, 2014, 15(71), doi: 10.1186/1129-2377-15-71.

[14] SCHER A.I., BIGAL M.E., LIPTON R.B. Comorbidity of migraine. Current Opinion in Neurology, 2005, 18, pp. 305-310.

[15] JENSEN R., STOVNER L.J. Epidemiology and comorbidity of headache. The Lancet Neurology, 2008, 7, pp. 354-361.

[16] SCHER A.I., TERWINDT G.M., PICAVET H.S., VERSCHUREN W.M., FERRARI M.D \& LAUNER L.J. Cardiovascular risk factors and migraine: the GEM population-based study. Neurology, 2005, 64, pp. 614-620.

[17] BigAl M.E., KURTH T., SANTANELlO N., BUSE D., GOLDEN W., ROBBINS M. \& LIPTON R.B. Migraine and cardiovascular disease: a population-based study. Neurology, 2010, 74, pp. 628-635

[18] MERIKANGAS K.R., ANGST J., ISLER H. Migraine and psychopathology. Results of the Zurich cohort study of young adults. Archives of General Psychiatry, 1990, 47, pp. 849-853.

[19] WANG S-J, FUH J-L, LU S.R., JUANG K.D. Quality of life differs among headache diagnoses: analysis of SF-36 survey in 901 headache patients. Pain, 2001, 89, pp. 285-292.

[20] MCWILliams L.A., GOODWIN R.D., COX B.J. Depression and anxiety associated with three pain conditions: results from a nationally representative sample. Pain, 2004, 111, pp. $77-83$.

[21] SMITHERMAN T.A., BASKIN S.M. Headache secondary to psychiatric disorders. Current Pain and Headache, 2008, 12, pp. 305-310.

[22] PEŇACOBA-PUENTE C., FERNÀNDES-DE-LAS-PEŇAS C., GONZÀLEZ-GUTIERREZ J.L., MIANGOLARRA-PAGE J.C., PAREJA J.A. Interaction Between anxiety, depression, quality of life and clinical parameters in chronic tension-type headache. European Journal of Pain, 2008, 12, pp. 886-894.

[23] MINEN M.T., TANEV K. Influence of psychiatric comorbidities in migraineurs in the emergency department. General Hospital Psychiatry, 2014, 36, pp. 533-538.

[24] SAUNDERS E.F., NAZIR R., KAMALI M., RYAN K.A., EVANS S., LANGENECKER S., GELENBERG A.J., MCINNIS M.G.Gender differences, clinical correlates, and longitudinal outcome of bipolar disorder with comorbid migraine. Journal of Clinical Psychiatry, 2014, 75, pp. 512-519.

[25] HAGEN K., EINARSEN C., ZWART J.A., SVEBAK S., BOVIM G. The co-occurrence of headache and musculoskeletal symptoms amongst 51,050 adults in Norway. European Journal of Neurology, 2002 9, pp. 527-533.

[26] MARCUS D.A., SCHARFF L., MERCER S., TURK, D.C. Musculoskeletal Abnormalities in Chronic Headache: A Controlled Comparison of Headache Diagnostic Groups. Headache, 1999, 39, pp. 21-27.

[27] SHARMA K., REMANAN R., SINGH S. Quality of life and psychiatric co-morbidity in Indian migraine patients: a headache clinic sample. Neurology India, 2013, 61, pp. 355-359. 


\section{Neural Network World 1/2020, 1-26}

[28] BABEL P. Memory of pain and affect associated with migraine and non-migraine headaches. Memory, 3, pp. 1-12, 2014.

[29] BUSCEMA P.M., MASSINI G., BREDA M., LODWICK W.A., NEWMAN F., ASADIZEYDABADI M. Artificial Adaptive Systems Using Auto Contractive Maps. Springer International Publishing, 2018, doi: 10.1007/978-3-319-75049-1.

[30] MCNAIR D.M., LORR M., DROPPLEMAN L.F. Profile of mood states. Educational and Industrial Testing Service Inc. San Diego, USA, 1981.

[31] BECK A., WARD C., MENDELSON M., MOCK J., ERBAUG, H. An Inventory for measuring depression. Archives of General Psychiatry, 1961, 4, pp. 561-571.

[32] BAGBY R.M., TAYLOR G.J.\& PARKER J.D. The Twenty-item Toronto Alexithymia ScaleII. Convergent, discriminant, and concurrent validity. Journal of Psychosomatic Research 1994, 38, pp. 33-40.

[33] SETTINERI S., MERLO E.M., PAGANO DRITTO I., Midili M., BRUNO A., MENTO C. Suppression mental questionnaire: A preliminary study. Mediterranean Journal of Clinical Psychology, 2016, 4(2).

[34] GAGEY P.M. Posturologia. Roma: Marrapese, 1997.

[35] BUSCEMA P.M., GROSSI E., SNOWDON D., ANTUONO P. Auto-Contractive Maps: an Artificial Adaptive System for Data Mining. An Application to Alzheimer Disease. Current Alzheimer Research, 2008, 5, pp. 481-498.

[36] COPPEDÈ F., GROSSI E., BUSCEMA P.M., MigLiORE L. Application of Artificial Neural Networks to Investigate One-Carbon Metabolism in Alzheimer's Disease and Healthy Matched Individuals. PlosOne, 2013, 8, pp. 1-11.

[37] BUSCEMA P.M., NEWMAN F., GROSSI E., TASTLE W. Application of Adaptive Systems Methodology to Radiotherapy. Paper presented at the NAFIPS (North American Fuzzy Information Processing Society) conference, Toronto, Canada, 2010, pp. 12-14.

[38] BUSCEMA P.M., PENCO S., GROSSI E. A Novel Mathematical Approach to Define the Genes/SNPs Conferring Risk or Protection in Sporadic Amyotrophic Lateral Sclerosis Based on Auto Contractive Map Neural Networks and Graph Theory. Neurology Research International, 2012, doi: 10.1155/2012/478560.

[39] COMPARE A., GROSSI, E., BUSCEMA P.M., ZARBO C., MAO X., FAlETRA F., PASOTTI E., MOCCETTI T., MOMMERSTEEG P.M.C., AURICCHIO A. Combining Personality Traits with Traditional Risk Factors for Coronary Stenosis: An Artificial Neural Networks Solution in Patients with Computed Tomography Detected Coronary Artery Disease. Cardiovascular Psychiatry and Neurology, doi: 10.1155/2013/814967.

[40] BUSCEMA P.M. A brief overview and introduction to Artificial Neural Networks. Substance Use, Misuse, Special Issue on The Middle Eastern Summer Institute on Drug Use. Proceedings: 1997/1999, 2002, 37, pp. 1093-1148.

[41] BUSCEMA P.M. A novel adapting mapping method for emergent properties discovery in databases: experience in medical field. Paper presented at the IEEE International Conference on Systems, Man and Cybernetics, Montreal, Canada, 7-10 October 2007.

[42] BUSCEMA P.M., GROSSI E. Artificial adaptive systems in medicine. New theories and models for application in the real world. Bentham E-Books - Bentham Science Publishers, 2009.

[43] International Headache Society, Headache Classification Subcommittee (2004) The International Classification of Headache disorders. $2^{\text {nd }}$ edition. Cephalalgia, 24 (S1), pp. 9-160.

[44] BUSCEMA P.M., MAZZETTI DI PIETRALATA M., SALVEMINI V., INTRALIGI M. \& INDRIMI M. Application of Artificial Neural Networks to Eating Disorders. Substance Use E Misuse, 1998, 33, pp. 765-791.

[45] BUSCEMA P.M. Squashing Theory and Contractive Map Network. Semeion Technical Paper, 32. Rome, 2007.

[46] BUSCEMA P.M., ASADI M., LODWICK W., BREDA M. The H0 function, a new index for detecting structural/topological complexity information in undirected graphs. Physica A: Statistical Mechanics and its Applications, 2016, 447, pp. 355-378. 
Gitto L. et al.: Affective symptoms and postural abnormalities as predictors...

\section{Appendix: a brief introduction to AutoCM and its graphical representation.}

AutoCM is useful in solving problems in which one wishes to find relationships among data, especially in partitioning data into clusters of related elements.

This version of AutoCM is unsupervised.

AutoCM has many features that are different from classic ANNs. They can be summarized as follows.

First, AutoCM processes all the variables together finding the many-to-many relationships among them; all its weights, before the training phase, are initialized with the same value (0.00000001) and not randomly. This means that AutoCM is a complete deterministic algorithm: processing the same data set we will get always the same results.

Then, AutoCM can treat both Boolean and real number data, also mixed in the same dataset. The data only needs to be linearly scaled, at the beginning, between 0 and 1.

AutoCM can be used both as an unsupervised ANN as well as its supervised version (K-CM); the supervised version of AutoCM (K-CM) will not show the phenomenon of over fitting during the training phase. At the end of the training phase, the AutoCM output is irrelevant since all the output vectors will be close to zero; instead, the weights, that at beginning were all initialized with the same value close to zero, will have been encoded, within themselves, the key information present into the dataset.

The final weights of a trained AutoCM are organized into a non-symmetric square matrix, where each variable shows a specific value of the nonlinear correlation with any other variable: all these associations can be expressed using fuzzy set theory; in addition, they can be projected in various forms of weighted, direct or indirect graphs.

AutoCM is not sensitive to the ratio between the number of variables, N, and number of records, P. It learns quickly and correctly when the number of variables, $\mathrm{N}$, is much bigger than the number of records $P(N \gg P)$, which means that AutoCM can easily learn both the given data set and its transposition. AutoCM can learn the many-to-many relationships between variables and records, projecting with the same metric into a weighted graph.

Moreover, the final matrix of weights of Auto-CM is a tensor matrix. In fact, if AutoCM learns a set of completely orthogonal input vectors, its final weights matrix will be all null except on the main diagonal. The final matrix of weights generated by AutoCM violates all the axioms of the concept of distance, its weights are asymmetric, they do not respect the triangular inequality, and the distance between a variable from itself may bigger than zero, thereby behaving as a matrix of tensors among the database variables.

The AutoCM learning process does not risk being trapped in local minima. The AutoCM trained weights may be used to generate a robust Markov machine defining the correct probability chains of transition between all the variables.

AutoCM provides a new type of energy minimization. 
While the usual minimization function is:

$$
E=\operatorname{Min}\left\{\sum_{i}^{N} \sum_{j}^{N} \sum_{q}^{M} x_{i}^{q} x_{j}^{q} \sigma_{i, j}\right\} ; \sigma_{i, j}>0,
$$

AutoCM minimizes the energy extracted from the data set according to the following equation:

$$
E=\operatorname{Min}\left\{\sum_{i}^{N} \sum_{j \neq i}^{N} \sum_{k \neq j \neq i}^{N} \sum_{q}^{M} x_{i}^{q} x_{j}^{q} x_{k}^{q} A_{i, j} A_{i, k}\right\},
$$

where $x_{i}^{q}=$ value of i-th input unit at $q$-th pattern;

$A_{i, j}=1-\frac{w_{i, j}}{C}$

$A_{i, k}=1-\frac{w_{i, k}}{C}$

$w_{i, j}<C, w_{i, k}<C$

$i j k=$ indices for the variables - columns;

$q=$ index for the patterns - rows;

$N=$ number of variables (columns);

$M=$ number of patterns (rows);

AutoCM, in many trials, has been shown to be the best algorithm able to detect weak and strong similarities among data.

The final AutoCM weights represented by its parameters can be used to construct a continuous hyper-surface of whole data set.

AutoCM belongs to any of the following three typological types classified as unsupervised ANNs:

a) Auto Poietic: AutoCM project each input vector in an expanded space of its weights;

b) Auto Associative: AutoCM weights define the hyper surface of the data set;

c) Dynamic Associative Memories (DAMs): AutoCM weights may be used for dynamic scenarios simulation.

To sum up, at the beginning of the training process with AutoCM, the input vector are repeated in the output layer (that memorizes the dataset); afterwards, each output vector is the union of all the input vectors of the data set (in this step the strong similarities are detected); in a third step, only the outputs that each input vector presents as different from the other input vectors are shown (i.e., the differences are detected); finally, at the end of the training, the outputs become null: there is a complete internalization of the data set into the weights.

Regarding the graphical representations of AutoCM, here are some more details about the Maximally Regular Graph (MRG) and the Minimum Spanning Tree (MST).

The MST represents the "nervous system" of any dataset, given by the sum of the strength of the connections among all the variables, that shows the total energy of that system. 
Gitto L. et al.: Affective symptoms and postural abnormalities as predictors...

The MST selects only the connections that minimize this energy. Consequently, all the links shown by MST are fundamental, but not every fundamental link of the dataset is shown by MST.

Such limit is intrinsic to the nature of MST itself: every link able to generate a cycle into the graph is eliminated, in spite of its strength. To prevent this limit and to explain the intrinsic complexity of a dataset, it is necessary to add more links to the graph, according to two criteria:

1) the new links have to be relevant from a quantitative point of view;

2) the new links have to be able to generate new cyclic regular microstructures, from a qualitative point of view.

Consequently, the MST Tree-graph is transformed into an undirected graph with cycles. Because of the cycles, the new graph is a dynamic system, including in its structure the time dimension. This is the reason why this new graph should provide information not only about the structure but also about the functions of the variables of the dataset.

To build this new graph we need to proceed in this way:

- assume the MST structure as a starting point of the new graph;

- consider the sorted list of the connections skipped during the MST generation;

- estimate the H Function of the new graph each time we add a new connection to the MST structure, to monitor the variation of the complexity of the new graph at every step.

The Maximally Regular Graph (MRG) [46] the graph whose H Function is the highest, among all the graphs generated adding to the original MST the new connections skipped before to complete the MST itself [29].

The MRG is given by the following equations:

$$
\begin{array}{ll}
H_{i}=f\left(G\left(A_{p}, N\right)\right) ; & \begin{array}{l}
\text { Generic Function on a graph with } \\
A_{p} \operatorname{arcs} e N \text { Nodes }
\end{array} \\
H_{i}=\frac{\mu_{p} \cdot \varphi_{p}-1}{A_{p}} ; & \begin{array}{l}
\text { Calculation of } H \text { Function, where } H_{0} \\
\text { represents MST complexity }
\end{array} \\
M R G=\operatorname{Max}\left\{H_{i}\right\} ; & \text { Graph with highest } H \\
i \in[0,1,2, \ldots, R] ; & \text { Index of } H \text { Function } \\
p \in[N-1, N, N+1, \ldots, N-1+R] ; & \text { Index for the number of graph arcs } \\
R \in\left[0,1, \ldots, \frac{(N-1) \cdot(N-2)}{2}\right] ; & \begin{array}{l}
\text { Number of the skipped arcs during } \\
\text { the M.S.T. generation }
\end{array}
\end{array}
$$

The " $R$ " variable is a key variable during the MRG generation. " $R$ ", in fact, could be also null, when the generation of MST implies no connections to be 
skipped. In this case, there is no MRG for that dataset. The " $R$ " variable, further, makes sure that the last, i.e., the weakest connection added to generate MRG is always more relevant that the weakest connection of MST.

The MRG, finally, generates the graph presenting the highest number of regular microstructures using the most important connections of the dataset. The highest the $\mathrm{H}$ Function selected to generate the MRG, the more meaningful the microstructures of the MRG itself.

(The authors suggest to see Buscema P.M., Massini G., Breda M., Lodwick W.A., Newman F., Asadi-Zeydabadi M. (2018). Artificial Adaptive Systems Using Auto Contractive Maps. Springer International Publishing, doi: 10.1007/ 978-3-319-75049-1 for further insights. The book is cited among the references [29]). 